\title{
High Energy Processes in Clusters of Galaxies and the Origin of Cosmic Rays
}

\author{
Xinyu Chi, ${ }^{1}$ Enoch C. M. Young, ${ }^{1}$ Peter K. N. Yu, ${ }^{1}$ Li Zhang, ${ }^{2}$ and Qing-Qi Zhu ${ }^{3}$ \\ ${ }^{1}$ Department of Physics and Materials Science, City University of Hong Kong, Hong Kong \\ ${ }^{2}$ Department of Physics, University of Hong Kong, Hong Kong \\ ${ }^{3}$ Institute of High Energy Physics, Chinese Academy of Sciences, Beijing 100039, China
}

(Received 17 April 1996)

\begin{abstract}
We test the hypothesis of a universal cosmic ray intensity by calculating the secondary electron or positron production in the hadronic interactions of cosmic ray nuclei with intergalactic gas within clusters of galaxies. We find that the spectral characteristics of the radio synchrotron emission by these secondary electrons is not consistent with observations of the Coma cluster. Thus the hypothesis can be ruled out on cluster scales. [S0031-9007(96)00983-0]

PACS numbers: 98.70.Sa, 13.85.Tp, 98.62.Ra, 98.70.Qy
\end{abstract}

The origin of cosmic rays was one of the great unsolved problems in modern astrophysics before the advent of the Compton Gamma Ray Observatory (CGRO) in the 1990s. The difficulty of acquiring information on their sources is well known - the tangled magnetic fields in the Milky Way Galaxy and in intergalactic space smear their arrival directions. Since high energy gamma rays $(>100 \mathrm{MeV})$ are produced in the interactions of cosmic rays with the interstellar medium, cosmic gamma ray experiments have been suggested as a way of testing the hypothesis of cosmic ray origin. One is a measurement of the GalactoCentric gradient of gamma ray emissivity [1] as there are more potential sources (supernova remnants) towards the Galactic Center. Another is the "Ginzburg test" [2] — a measurement of gamma rays from the Large Magellanic Cloud where there is a large amount of atomic hydrogen gas acting as a target for extragalactic cosmic rays. Using the measurements of high energy gamma ray fluxes by the EGRET instrument on board CGRO, the Ginzburg test was performed for the Large Magellanic Cloud [3] and for the Small Magellanic Cloud [4], respectively. These two analyses unanimously concluded that the cosmic ray intensities within these galaxies are far below the local galactic value and thus ruled out the hypothesis of a universal cosmic ray intensity, at least in the local group of galaxies.

However, Dar and Shaviv [5] have recently argued that the cosmic ray intensity may vary within galaxies, groups, and clusters due to local magnetic fields and the distributions of sources. The results from the above Ginzburg tests may thus be caused by the shielding effect of the local magnetic field on these two galaxies so as to exclude cosmic rays. They suggest that the average cosmic ray intensity within clusters of galaxies be still high and approximately equal to that observed locally in our Galaxy. A consequence from this hypothesis is that the high energy gamma rays produced by the universal cosmic rays in clusters could account for the extragalactic diffuse background, and that some gas-rich nearby clusters, such as Coma, Perseus, and Virgo, are marginally visible to the EGRET instrument. A similar conclusion was reached by Said et al. [6] more than one decade ago, but the available information on the gas distribution in clusters of galaxies was poor at that time.

This version of an extragalactic origin of cosmic rays certainly has some merit and deserves further study. As it has been known for more than two decades, active galaxies are more powerful than our Galaxy in producing cosmic rays [7] and since many rich clusters contain active galaxies, the average cosmic ray intensity within the clusters could be substantially higher than that in the local group of galaxies. In this Letter, we test the hypothesis by calculating the intensity of secondary electrons (both $e^{+}$and $e^{-}$) produced in the interactions of cosmic rays with intergalactic gas and the radio emission by these secondary electrons, then comparing the emissions with observational data.

Diffusive shock acceleration is generally accepted as the working mechanism for generation of the bulk of the galactic cosmic rays by supernova remnants [8]. Similar acceleration processes are believed to occur in the lobes of radio galaxies and active galactic nuclei [9]. The shock-accelerated particles have a power-law spectrum in rigidity and the spectral index is 2 for strong shocks. The ambient galactic cosmic rays are observed as having a steeper power-law spectrum due to the rigidity-dependent diffusion which also leads to the escape from the Galaxy. It is easy to show if the spectral index at injection is $\alpha$ and the power-law index of the diffusion coefficient in the interstellar medium is $\delta$, then the spectral index of the ambient cosmic rays is $\alpha+\delta$ for the case of diffusion being the sole propagation effect. However, the cosmic rays that escape (from the Galaxy by diffusion) have the same spectral index as that at injection. The cosmic rays (protons) in intergalactic space within clusters can either come from galaxies or be directly accelerated in situ by intergalactic shocks [10]. If we treat each cluster as a "closed box" for cosmic rays in this work, their spectrum can be taken as a power law $K E^{-2.0}$, where $E$ is the particle energy and $K$ is a constant. This spectrum is flatter than that adopted by Dar and Shaviv [5], that is, $K E^{-2.7}$. 
In the hadronic collisions of protons on protons, a large number of pions are created via inelastic processes. Because of the involvement of the strong force, accurate calculations of the inelastic cross section and multiplicity have not been possible so far, particularly for the soft processes involved. Empirical approaches are usually adopted for the formulations. Here we use all the available data from accelerator experiments to derive the energy dependences of cross section and multiplicity. For the $p-p$ inelastic collisions, we fit the data [11] in the energy range $25-1.7 \times 10^{6} \mathrm{GeV}$ in the laboratory frame. The cross section is derived as a power law of particle energy in the laboratory frame

$$
\sigma=25.7 E_{p}^{0.08} \mathrm{mb}
$$

where the proton energy $E_{p}$ is in units of $\mathrm{GeV}$. A similar fit to the charged particle multiplicity data [12] in the energy range $25-1.6 \times 10^{5} \mathrm{GeV}$ leads to

$$
\xi_{\pi^{ \pm}}=2.25 E_{p}^{0.22} \text {. }
$$

For simplicity of calculation, we use the monoenergetic approximation for the pions generated by a fixed $E_{p}$. The average energy of the pions can be expressed in terms of $E_{p}$,

$$
\left\langle E_{\pi}\right\rangle\left(E_{p}\right)=\frac{2}{3} \frac{\kappa E_{p}}{\xi} \simeq \frac{E_{p}^{0.78}}{6.75} \mathrm{GeV},
$$

where $\kappa=1 / 2$ is the mean inelasticity, and the factor $2 / 3$ comes from the isospin symmetry, i.e., $2 \xi\left(\pi^{0}\right)=$ $\xi\left(\pi^{+}\right)+\xi\left(\pi^{-}\right)$. The charged pion source function for $p$ - $p$ collisions above the threshold $E_{\mathrm{th}}=1.22 \mathrm{GeV}$ can be derived with Eqs. (1)-(3), as follows:

$$
\begin{aligned}
q_{\pi}\left(E_{\pi}\right) & =c n_{\mathrm{H}} n_{p}\left(E_{p}\right) \xi_{\pi^{ \pm}}\left(E_{p}\right) \sigma\left(E_{p}\right) \frac{d E_{p}}{d E_{\pi}} \\
& =1.34 \times 10^{-26} K c n_{\mathrm{H}} E_{\pi}^{-1.90} \mathrm{~cm}^{-3} \mathrm{~s}^{-1} \mathrm{GeV}^{-1},
\end{aligned}
$$

where $c$ is the speed of light, $n_{\mathrm{H}}$ is the number density of target hydrogen, and $n_{p}=K E_{p}^{-2.0}$ is the differential number density of cosmic ray protons.

The production of secondary electrons through the decay reactions $\pi^{ \pm} \rightarrow \mu^{ \pm} \rightarrow e^{ \pm}$has been calculated in detail [13]. In the laboratory frame, the mean electron energy is a quarter of the pion energy, and the electron source function is obtained as

$$
\begin{aligned}
q_{e}\left(E_{e}\right) & =q_{\pi}\left(E_{\pi}\right) \frac{d E_{\pi}}{d E_{e}} \\
& =3.85 \times 10^{-27} K c n_{\mathrm{H}} E_{e}^{-1.90} \mathrm{~cm}^{-3} \mathrm{~s}^{-1} \mathrm{GeV}^{-1}
\end{aligned}
$$

The electron energy range is determined by the threshold energy $\left(E_{\mathrm{th}}=1.22 \mathrm{GeV}\right)$ for projectile protons for the lower limit and by the high energy cutoff in the proton spectrum for the upper limit. The lower limit can be determined by experiment of positive muon decays. The result indicates that the most probable electron energy occurs at half of the muon mass, i.e., $53 \mathrm{MeV}$ [14]. There is also an upper limit if the cosmic ray proton spectrum has a cutoff at $10^{14} \mathrm{eV}$ as commonly adopted for shock acceleration in supernova remnants. The estimate of this upper limit is rather more complicated as there are two or three pions carrying half of the total inelastic energy. Including this effect, we made a conservative estimate for the upper limit value to be about $180 \mathrm{GeV}$.

When applying the above formulation to electron production in clusters of galaxies, the chemical compositions of cosmic rays and intergalactic gas can be effectively taken into account by multiplying two factors to the above source function, 1.3 for cosmic rays and 1.26 for the intergalactic medium. In the environment of clusters, the main process for the energy loss of the electrons is inverse Compton scattering with the cosmic microwave background (CMB) photons. Since here we consider only the nearby clusters, we can neglect the effect of cosmological evolution on the CMB photons. Let us define the ambient density of the electrons to be $n_{e}$, then it can be derived from the transport equation

$$
-\frac{\partial}{\partial E_{e}}\left(\dot{E}_{e} n_{e}\right)=q_{e}\left(E_{e}\right)
$$

where $\dot{E}_{e}=2.5 \times 10^{-17} E_{e}^{2} \mathrm{GeV} \mathrm{s}^{-1}$ is the inverse Compton energy loss rate in the CMB of energy density $0.24 \mathrm{eV} \mathrm{cm}^{-3}$, from the Bethe-Heitler formula [15]. Integrating the equation over $E_{e}$, we obtain the solution

$$
\begin{aligned}
n_{e}= & 2.53 \times 10^{-10} K_{c n} E_{e}^{-2.0} \\
& \times\left(E_{e}^{-0.90}-E_{e, u}^{-0.90}\right) \mathrm{cm}^{-3} \mathrm{GeV}^{-1},
\end{aligned}
$$

where $E_{e, u}=180 \mathrm{GeV}$ is the upper bound of the injection spectrum.

The numerical value of the electron density can be obtained by normalizing the equilibrium intensity of cosmic ray nuclei in clusters to that of observed locally. Above $2.5 \mathrm{GeV} /$ nucleon, the local cosmic ray (nuclei) intensity after demodulation [16] is 0.138 nucleon ${ }^{-1} \mathrm{~cm}^{-2} \mathrm{~s}^{-1} \mathrm{sr}^{-1}$, thus $K=1.47 \times 10^{-10} \mathrm{~cm}^{-3} \mathrm{GeV}$. Another important parameter, $n_{\mathrm{H}}$, can be derived from x-ray emission. For the Coma cluster, $n_{\mathrm{H}}$ is found to be a function of the radial distance from the center of the cluster [17]. It varies from $3.0 \times 10^{-3}$ at the cluster center to $2.0 \times 10^{-4} \mathrm{~cm}^{-3}$ at angular distance $40^{\prime}$. Inserting $K$ and $n_{\mathrm{H}}$ into the above equation, we have

$$
\begin{aligned}
n_{e}= & 3.35 \times 10^{-12}\left(\frac{n_{\mathrm{H}}}{3.0 \times 10^{-3} \mathrm{~cm}^{-3}}\right) \\
& \times E_{e}^{-2.0}\left(E_{e}^{-0.90}-E_{e, u}^{-0.90}\right) \mathrm{cm}^{-3} \mathrm{GeV}^{-1} .
\end{aligned}
$$

In the $\mathrm{GeV}$ energy range where radio synchrotron emission comes from, the spectrum can be well approximated by a power law with index 2.90 . 
The halo around the center of the Coma cluster of galaxies has been surveyed in radio emission at many wavelengths. While our predicted electron intensity is sufficient for reproducing the observed radio flux [18] via synchrotron radiation, the spectral index is different. The observed index is estimated to be $1.34 \pm 0.06$ in the frequency range $10 \mathrm{MHz}-1.4 \mathrm{GHz}$, but the prediction by the synchrotron radiation formula [19] from Eq. (8) is only 0.95 . Furthermore, the observed index varies with the distance from the cluster center [20]. At the bridge between the Coma cluster and Abell 1367 which is about $2 \mathrm{Mpc}$ from the Coma center, it becomes even steeper, about 1.5. This spectral difference indicates that the bulk of relativistic electrons responsible for the radio emission does not originate from the interactions of cosmic rays with intergalactic gas as we modeled above, rather from the individual galaxies within the cluster. On their way to the intergalactic space, they lose energies and their energy spectrum steepens. A similar spatial variation of the radio spectrum has been observed in the Perseus cluster [21]. In summary, the existence of a universal intensity of cosmic rays which is comparable to that locally observed can now be ruled out on the cluster scale and even on the supercluster scale. The cosmic ray intensity within clusters is indeed significantly lower than that observed in the solar vicinity.

An independent test of the hypothesis has been made by Stecker and Salamon [22]. These authors refute it by using a mismatch argument with the gamma ray spectra. The measured Diffuse Gamma Ray Background by EGRET is a flat power law, possibly with a "concave" feature, whereas the neutral pion decay spectrum is steep and "convex" featured. Such a test is universal in the sense that observed gamma ray background is averaged over galaxies, groups, and clusters. Therefore the local variation argument of Dar and Shaviv can be dissolved.

Another possible test of the hypothesis of a universal cosmic ray intensity is to look at the high energy $(>100 \mathrm{MeV})$ gamma ray fluxes from the nearby clusters, as detailed by Dar and Shaviv [5]. Unfortunately, the current gamma ray instrument EGRET on board the CGRO has a marginal sensitivity for these sources if the hypothesis holds and so far the result has been null [23]. One has to wait for the next generation of experiments. A further test for the hypothesis can also be made by observations of low energy gamma rays. As mentioned earlier, the main energy loss for the secondary electrons is the inverse Compton scattering with CMB photons, and consequently the radiation energy will largely be converted into low energy gamma rays. For the Coma cluster, we estimate that the gamma ray flux in the energy range $50-200 \mathrm{keV}$ be $\sim 10^{-5} \mathrm{~cm}^{-2} \mathrm{~s}^{-1}$. Although the current BATSE and OSSE experiments on CGRO are not sensitive to this flux level, the future generation of experiments will fulfill the task. A result of nondetection from all the experiments will support the conclusions given here.

The authors wish to thank Professor Sir Arnold Wolfendale for useful communication. This work is supported by the Strategic Fund of the City University of Hong Kong.

[1] D. Dodds, A. W. Strong, and A. W. Wolfendale, Mon. Not. R. Astron. Soc. 171, 569 (1976).

[2] V.L. Ginzburg, Nature Phys. Sci. Suppl. 239, 8 (1972).

[3] X. Chi and A. W. Wolfendale, J. Phys. G 19, 795 (1993).

[4] P. Sreekumar et al., Phys. Rev. Lett. 70, 127 (1993).

[5] A. Dar and N. Shaviv, Phys. Rev. Lett. 75, 3052 (1995).

[6] S. S. Said, A. W. Wolfendale, M. Giller, and J. Wdowczyk, J. Phys. G 8, 383 (1982).

[7] G. R. Burbidge, Philos. Trans. R. Soc. London A 277, 481 (1975).

[8] For review, W. I. Axford, Astrophys. J. Suppl. 90, 937 (1994).

[9] For instance, W. I. Axford, Ann. N.Y. Acad. Sci. 375, 297 (1981); P.L. Biermann and P. A. Strittmatter, Astrophys. J. 322, 643 (1987).

[10] C. A. Norman, D. B. Melrose, and A. Achterberg, Astrophys. J. 454, 60 (1995).

[11] Particle Data Group, Phys. Rev. D 50, 1173 (1994), and references therein.

[12] UA5 Collaboration, Phys. Rep. 154, 247 (1987).

[13] J. H. Scanlon and S. N. Milford, Astrophys. J. 141, 718 (1965); R. Ramaty and R.E. Lingenfelter, J. Geophys. Res. 71, 3687 (1966); G. C. Perola, L. Scarsi, and G. Sironi, Nuovo Cimento B 52, 455 (1967).

[14] M. Bardon et al., Phys. Rev. Lett. 14, 449 (1965).

[15] W. Heitler, The Quantum Theory of Radiation (Oxford Press, London, 1960), 2nd ed.

[16] B. A. Randall and J. A. van Allen, Geophys. Lett. 13, 628 (1986).

[17] J. P. Hughes, P. Gorenstein, and D. Fabricant, Astrophys. J. 329, 82 (1988).

[18] K.-T. Kim, P.P. Krongberg, P.E. Dewdney, and T.L. Landecker, Astrophys. J. 355, 29 (1990).

[19] V.L. Ginzburg and S. I. Syrovatskii, The Origin of Cosmic Rays (Pergamon Press, Oxford, 1964).

[20] K.-T. Kim, P.P. Krongberg, G. Giovannini, and T. Venturi, Nature (London) 341, 720 (1989).

[21] A. Pedler et al., Mon. Not. R. Astron. Soc. 246, 477 (1990).

[22] F. W. Stecker and M. H. Salamon, Phys. Rev. Lett. 76, 3878 (1996).

[23] P. Sreekumar et al., Astrophys. J. 464, 628 (1996). 